\title{
Effect of Lifestyle Modification Intervention on Health Status of Coronary Artery Disease Patients: Randomized Control Trial
}

\author{
Manal Hamed Mahmoud ${ }^{1} \&$ Sabah Nazeh Elderiny ${ }^{2}$ \\ ${ }^{1}$ Medical-Surgical Nursing, Faculty of Nursing, Benha University, Egypt \\ ${ }^{2}$ Medical-Surgical Nursing, Faculty of Nursing, Helwan University, Egypt \\ Correspondence: Manal Hamed Mahmoud, Assistant Professor of Medical-Surgical Nursing, Faculty of Nursing, \\ Benha University, Egypt.
}

Received: October 15, 2018

Accepted: December 12, 2018

Online Published: December 19, 2018

doi:10.20849/ijsn.v3i3.523

URL: https://doi.org/10.20849/ijsn.v3i3.523

\begin{abstract}
Background: Coronary artery disease is a major cause of morbidity and mortality throughout the world. Modification of life style can be achieved by patients' education to improve their health status. Aim: To evaluate effect of lifestyle modification intervention on health status of coronary artery disease patients. Design: Randomized control trial used to achieve the aim of this study. Setting: The current study conducted at the coronary care unit and cardiology out-patient clinics of Benha University Hospital, Benha City. Subjects: Purposive sample of 92 coronary artery disease patients (study and control) were included in the study. Tools: Two tools used in this study 1) Interview questionnaire. 2) Health status questionnaire. Results: Significant percentage of the study participants from both groups were adhered to unhealthy life-style practices as smoking, eating salty and fatty foods, drinking tea and coffee as well as they didn't practice exercises and recreational activities. After lifestyle modification intervention, there were significant statistical differences between the two groups favoring the study group regarding all aspects of health status; physical function, bodily pain, social function, role limitation due to physical or emotional problems and mental health $(\mathrm{P}<0.05)$. Conclusion and recommendations: This study concluded that life style modification intervention for coronary artery disease patients is effective and useful for enhancing their health status. This study recommended that coronary artery patients` education should be held in permanent approach, because it is a crucial part of their treatment.
\end{abstract}

Keywords: coronary artery disease, health status, intervention, lifestyle modification

\section{Introduction}

Cardiovascular disease is a main cause of mortality and disability in spite of extensive hard work to manage cardiac risk factors in the course of diet, exercise, and medications (Roger, et al 2012). Cardiovascular disease (CVD) comprises all diseases of the circulatory system, cerebro-vascular disease, hypertension, peripheral arterial disease, rheumatic heart disease, congenital disease, heart failure, deep vein thrombosis and pulmonary embolism and coronary heart disease (Go, et al, 2014). Coronary artery disease (CAD), as well referred to as coronary heart disease (CHD), is the tapering of the small blood vessels that deliver blood and oxygen to the heart (United States National Library of Medicine, 2016). Coronary artery disease (CAD) incorporates acute myocardial infarction, other acute ischemic heart disease, angina pectoris, atherosclerotic cardiovascular disease, and all other types of chronic ischemic coronary heart disease (Go, et al, 2014).

The cardiovascular risk factors are classified as non-modifiable or modifiable risk factors. Non-modifiable risk factors comprise age, sex, family history, and having a prior heart attack. Modifiable risk factors are hypertension, hyperlipidemia, diabetes mellitus, and smoking (Framingham, 2016). Clinical data verified that a sedentary lifestyle, stress and fatty diet are important modifiable risk factor for CAD (Weberg, 2013). Health care workers didn't succeed to modify mainly preventable (modifiable) risk factors and the crisis of cardiovascular risk and disease incidence subsists due to poor management of modifiable risk factors (Wachira, \& Stys, 2013). There are numerous clinical strategies that suggested lifestyle modifications for the prevention and the treatment of CVDs which included complete smoking cessation, blood pressure control, lipid management, physical activity, weight management, type two diabetes mellitus management, medication, and cardiac rehabilitation (Smith et al, 2011). A healthy diet, regular exercise, avoiding smoking, depression and further psychosocial activities, offer reimbursement to patients with CAD so patient education about lifestyle 
modification, execution approaches and follow up on a continuing foundation to achieve secondary prevention. Successful coronary revascularization and treatment ought to be accomplished by superior labors to recognize and modify risk factors and their original causes (Brinks et al, 2017).

Providing sufficient knowledge about CAD through cardiac educational program is supposed to be an essential obligation that enhanced knowledge, awareness, attitudes, and beliefs for compliance to healthy lifestyle (Eshah, Bond, \&, Froelicher, 2010). Cardiac patient education is a vital element of nursing care aimed at supporting those patients to take care of themselves (Fredericks et al, 2010) Nurses have a chief responsibility to instruct, assist and guide patients to create lifestyle changes. Nurses are capable of providing professional information and assistance in either a counseling or rehabilitation setting via a holistic approach to care. Smoking termination, relaxation techniques and stress management can be carried out with the patient and his/her health care provider in a relaxed, familiar manner (Coster, \& Norman, 2009).

\subsection{Significance of the Study}

According to WHO statistics, about 17.7 million people die each year from CVDs, an estimated $31 \%$ of all deaths all over the world. Most of CVD deaths occur in low-income and middle-income countries, while $80 \%$ of all CVD deaths are caused by coronary heart disease and strokes. It is estimated that $54 \%$ of deaths from non-communicable diseases in the Eastern Mediterranean Region are due to cardiovascular diseases. The Region has higher rates of physical inactivity than other regions (World Health Organization, 2017).

Coronary heart disease deaths in Egypt reached 107,232 or $23.14 \%$ of total deaths. The age adjusted death rate is 186.36 per 100,000 of population ranks Egypt as the 23 in the world (World Health Organization, 2014). Triggering coronary artery diseases are smoking, unhealthy diet, physical inactivity and the hurtful use of alcohol. These in turn show up in people as elevated blood pressure, hyperglycemia, overweight and obesity, risks injurious to good quality heart health (World Health Organization, 2017). Great quantity of deaths can be reduced by modifying risk factors and obedience to healthy life style and this concern need extreme effort to progress knowledge of patients (Ahmad, A., Tawalbeh, 2015). For this reason the researchers conducted this study to help those patients to adhere to healthy lifestyle to enhance their health status.

\section{Aim of the Study}

This study aimed to evaluate effect of lifestyle modification intervention on health status of coronary artery disease patients through:

Assess patients' health status and its related variables.

Develop and implement life style modification intervention for the study group.

Evaluate effect of life style modification intervention on health status of coronary artery disease patients (study group).

\subsection{Research Hypothesis}

Health status among the study group will be enhanced after execution of lifestyle modification intervention on health status compared to control group.

\section{Subjects and Methods}

Research design: randomized control trial used to achieve the aim of this study.

\subsection{Setting}

The current study conducted at the coronary care unit (CCU) and cardiology out-patient clinics of Benha University Hospital, Benha city. The coronary care unit consists of two rooms, the big one contains eight beds and the small room included four beds. Both rooms are equipped by all necessary devices and equipment needed for care of coronary artery patients.

\subsection{Sample}

A purposive sample of 92 coronary artery patients (angina and MI) included in this study. Patient with other chronic disorders were excluded from the study. Sample size determined based on a previous study reported that health status score was significantly improved in the study intervention group from $4.8 \pm 1.0$ to $5.1 \pm 0.9$ (Lourenço et al., 2015). Considering level of significance of 5\%, and power of study of $80 \%$, the sample size can be calculated using the following formula:

$\mathrm{n}=\left[\left(\mathrm{Z}_{\alpha / 2}+\mathrm{Z}_{\beta}\right)^{2} \times\left\{2(\mathrm{SD})^{2}\right\}\right] /(\text { Difference })^{2}$

Therefore, $\mathrm{n}=\left[(1.96+0.84)^{2} \times\left\{2(0.9)^{2}\right\}\right] /(0.53)^{2}=45.2$. 
Based on the above formula, the sample size required per group is 46 .

\subsection{Tools of Data Collection}

Two tools utilized for data collection in the current study.

\subsubsection{Tool I}

Interview questionnaire; it involved two parts:

Part 1: Socio-demographic data of the study participants.

Included information about study participants' age, gender, marital status, residence, educational level and occupation.

Part 2: Assessment of lifestyle practices; It was developed by the researchers after reviewing of related literature (Timby \& Smith 2010 and Libby et al. 2008). It is a likert like scale with five options; repeatedly, often occasionally, rarely and never to assess lifestyle practices among the study participants. It included practices of smoking; (three items) practicing cigarette smoking, other types of tobacco used and daily intensity of smoking practices, eating unhealthy foods; (five items) eating fatty foods, eating fast foods, eating frying foods, eating salty foods and eating canned foods. Having unhealthy drinks; (three items) drinking coffee, tea and fatty drinks and practicing activities (three items) practice exercises, walking and recreational activity.

Scoring system: for negative life style practices (smoking, eating unhealthy foods and having unhealthy drinks) repeatedly ( 1 score), often ( 2 scores), occasionally (3 scores), rarely (4 scores) and never ( 5 scores) for each item, while for positive lifestyle practices (practicing activities, repeatedly ( 5 score), often (4 scores), occasionally ( 3 scores), rarely (2 scores) and never (1 score) for each item.

Scores for smoking ranged from 3 to 15 .

Scores for eating unhealthy foods ranged from 5 to 25 .

Scores for having unhealthy drinks ranged from 3 to 15 .

Scores for practicing activities ranged from 3 to 15 .

Total lifestyle practices scores ranged from 14 to 70 , and classifies as the following:

Scores from 14 to $<30$ reflects poor lifestyle practices

Scores from 30 to $<50$ reflects fair lifestyle practices

Scores $\geq 50$ or more reflects good lifestyle practices

\subsubsection{Tool II}

Health status questionnaire: adapted from Ware and Sherbourne (1992). It is an instrument for measuring health status and outcomes from the patient's point of view, measuring eight dimensions; physical function, role limitation caused by physical problems, role limitations caused by emotional problems, mental health, energy/fatigue, bodily pain, social function and general health perception. It consists of five parts (scales):

Part 1: Physical function consisted of 10 items distributed on a scale of three options; yes, limited a lot (0 mark), yes, limited a little (50 mark) and no not limited at all (100 mark).

Part 2: Comprised role limitation caused by physical problems ( 3 items) and role limitations caused by emotional problems (3 items), both distributed on a scale of two options; no (0 mark) and yes (100 mark).

Part 3: Included mental health (5 items) and energy/fatigue (4 items), both spread on a scale of six options, all of the time ( 0 mark), most of the time (20 mark), a good bit of the time (40 mark), a little of the time (60 mark), some of the time ( 80 mark) and non of the time (100 mark).

Part 4: Contained bodily pain ( 2 items) and social function ( 2 items), both dispersed on a scale of five options; extremely (0 mark), quite a bit (25 mark), moderately (50 mark), a little bit (75 mark) and not at all (100 mark).

Part 5: General health perception; included 4 items distributed on a scale of five options; definitely true (0 mark), mostly true (25mark), don't know (50 mark), mostly false (27 mark) and definitely false (100 mark).

\subsubsection{Scoring System}

The Health status questionnaire is divided into five scales, each item in each scale is scored on a 0 to 100 range so that the lowest and highest possible scores are 0 and 100, respectively. Scores represent the percentage of total possible score achieved, items averaged together to create each scale. Total health status scores were categorized as the following: 
Scores from 0 to $<40$ reflects poor health status

Scores from 40 to $<60$ reflects fair health status

Scores $\geq 60$ reflects good health status

\subsection{Content Validity}

Tools were evaluated by three experts in medical-surgical nursing and two cardiologists tested the content validity. The tools and booklet adapted according to the experts' suggestion.

\subsection{Tools Reliability}

Reliability of tool I was tested through a pilot study by using Cronbach's $\alpha$ (alpha $=0.73$ ) for tool II, and $\alpha$ (alpha $=0.85$ ) for tool II, hence the questionnaire was found to be highly reliable.

\subsection{Ethical Considerations}

An ethical approval was attained from the Dean of Faculty of Nursing at Benha University to apply this study following explaining its aim. An informed written consent was gained from the study's participants and they were informed about their optional involvement in the study and that they have the right to withdraw from the study at any time. Participants assured that their rejoinder would be covert and only cumulative data would be conversed. After the study was fulfilled, handout about life-style modification was dispersed to the control group.

\subsection{Pilot Study}

A pilot study conducted on $10 \%$ of the study subjects' in order to assess the suitability and implication of the study tools and to test clarity of the questionnaire as well as to estimate the time needed to answer them; and then the essential modification done and these subjects excluded from the study sample.

\subsection{Field Work}

A written official conformity was obtained from the Director of Benha University Hospital and the head of coronary care unit to carry out the study after clarification of its purpose. It was conducted through four phases: assessment, planning, implementation, and evaluation. These phases were executed over eight months from the beginning of December 2017 to the end of July 2018.

\subsubsection{Assessment Phase}

After obtaining official agreement for study conduction, the researchers interviewed the coronary artery patients of both groups to explain the aim and procedures of the study and solicit for their cooperative contribution and written consents obtained from them and assured that data will be confidentially secured and they have the right to withdraw from the study at any time. Subjects were interviewed individually to assess their sociodemographic characteristics, assess their lifestyle practices and assess their health status by the use of the study tools. The data was obtained during this phase represented the baseline for further comparisons to assess the effect of the intervention. The control group was assessed firstly to prevent cross contamination of data.

\subsubsection{Planning and Implementation Phase}

Based on the requirements recognized in the assessment phase from the study group and in view of the allied literature, the researchers established a power point presentation about healthy life style for coronary artery patients with simple arabic language to suit patients' level of comprehension, as well as demonstration of procedures as coughing and breathing exercises. It insisted on the regions of chief deficit in patients' knowledge and unhealthy habits which included definition, incidence, risk factors, signs and symptoms, management measures, healthy foods, healthy eating habits, practicing exercises, activities and weight reduction, as well as blood pressure control, smoking cessation and stress management. The educational intervention involved five sessions that were conducted on small group (8-10) of the study group in out-patient clinics. It was achieved according to participants' physical and mental willingness. The duration of each session lasted from 20-30 minutes including periods of discussion according to their understanding, progress and feedback. Different methods of teaching were used such as lecture, power point, group discussion and brainstorming as well as a booklet containing all illustrations about health style modification with pictures was given to study group.

\subsubsection{Evaluation Phase}

After the educational intervention; its effect was evaluated by using the same health status questionnaire of pre-test after four months of intervention using post-test which was conducted for the control group first then the study group. 


\subsection{Statistical Analysis}

All statistical analyses were performed using SPSS for windows version 20.0 (SPSS, Chicago, IL). Data were tested for normality of distribution prior to any calculations. Continuous data were expressed in mean \pm standard deviation (SD) while categorical data were expressed in number and percentage. Chi-square test was used for comparison of variables with categorical data. Statistical significance was set at $\mathrm{p}<0.05$.

\section{Results}

Table 1 shows that, there was no statistically significant difference between socio-demographic characteristics within the two groups $(\mathrm{P}>0.05)$. More than half of the study and control groups were females (56.5 \& 52.2), while half of the study group and more than half of the control group lived in rural area (50\% \& $56.5 \%)$ and also more than half of them were working $(58.7 \% \& 63 \%)$ respectively. The majority of the study and control groups were married $(78.3 \% \& 80.4 \%)$, while only about one third of them were read and write $(37 \% \& 34.8 \%)$ respectively.

Figure 1 illustrates that, more than half of the studied patients had 50-69 and 60-69 years (26.1 \& 34.8\%) respectively, while in control group there was about two thirds of the patients had 50-69 and 60-69 years of age with $(32.6 \% \& 32.6 \%)$ respectively.

Figure 2 shows that, distribution of diagnosis within the two groups, high prevalence of angina pectoris than MI within control and study groups were $(76.1 \%, 69.6 \%)$ respectively.

Table 2 represents that mean score of total lifestyle practices among the studied patients were $(43.1 \pm 9.2 \& 40.3$ \pm 8.2 ) for the study and control group respectively which fair lifestyle practices. Table 3 shows that, there was no statistically significant difference between the two groups before intervention regarding physical function ( $P>0.05)$, while there was statistically significant difference after four months of intervention between them regarding all aspects of physical function $(\mathrm{P}<0.05)$. Additionally, there was no statistically significant difference between pre and post intervention within the control group, while there was statistically significant difference pre and post intervention within the study group.

Table 4 represents that, there was no statistically significant difference between the two groups pre intervention regarding role limitation $(\mathrm{P}>0.05)$, due to physical problems, bodily pain and social function while there was statistically significant difference after four months of intervention regarding role limitation $(\mathrm{P}<0.05)$. Furthermore, there was no statistically significant difference between pre and post intervention within the control group, while there was statistically significant difference pre and post intervention within the study group.

Table 5 demonstrates that, there was no statistically significant difference between the two groups before intervention regarding mental health and role limitations caused by emotional problems $(P>0.05)$, while there was statistically significant difference after four months of intervention between them $(\mathrm{P}<0.05)$. In addition, there was no statistically significant difference between pre and post intervention within the control group, while there was statistically significant difference pre and post intervention within the study group.

Table 6 explains that, there was no statistically significant difference between the two groups before guidelines intervention regarding energy/fatigue and general health $(P>0.05)$, while there was statistically significant difference after four months of intervention between them $(\mathrm{P}<0.05)$. Furthermore, there was no statistically significant difference between pre and post intervention within the control group, while there was statistically significant difference pre and post intervention guidelines within the study group.

Table 7 presents that, there was no statistically significant difference between the two groups before intervention regarding health status $(\mathrm{P}>0.05)$, while there was statistically significant difference after four months of intervention between them $(\mathrm{P}<0.05)$. Additionally, there was no statistically significant difference between pre and post intervention within the control group, while there was statistically significant difference pre and post intervention within the study group. Total health status score for the study and control group Mean \pm SD (46.9 $\pm 18.1 \& 45.9 \pm 16.6)$ which reflects fair health status pre intervention, while it improved to $(63.2 \pm 14.5)$ for the intervention group after 4 months of intervention that reflects good health status. 
Table 1. Socio- demographic characteristics of the studied patients with in the two group (n=92)

\begin{tabular}{llllllll}
\hline Socio-demographic data & & \multicolumn{2}{c}{ Study group } & \multicolumn{2}{c}{ Control group } & \multicolumn{2}{c}{ Chi square test } \\
& & $\mathrm{n}$ & $\%$ & $\mathrm{n}$ & $\%$ & $\chi^{2}$ & P value \\
\hline Age (years) & Mean \pm SD & $55.9 \pm 9.7$ & \multicolumn{2}{c}{$55.5 \pm 9.3$} & 0.164 & 0.870 \\
Gender & Male & 20 & 43.5 & 22 & 47.8 & & \\
& Female & 26 & 56.5 & 24 & 52.2 & 0.175 & 0.675 \\
Residence & Rural & 23 & 50.0 & 26 & 56.5 & & \\
& Urban & 23 & 50.0 & 20 & 43.5 & 0.393 & 0.531 \\
& Married & 36 & 78.3 & 37 & 80.4 & & \\
Marital status & Single & 4 & 8.7 & 4 & 8.7 & 0.105 & 0.949 \\
& Divorced/widow & 6 & 13.0 & 5 & 10.9 & & \\
Working status & Working & 27 & 58.7 & 29 & 63.0 & & \\
& Not working & 19 & 41.3 & 17 & 37.0 & 0.183 & 0.669 \\
& Illiterate & 16 & 34.8 & 15 & 32.6 & & \\
Educational level & Read/write & 17 & 37.0 & 16 & 34.8 & 0.313 & 0.958 \\
& Secondary & 7 & 15.2 & 9 & 19.6 & & \\
\hline
\end{tabular}

Significant at $P \leq 0.05$

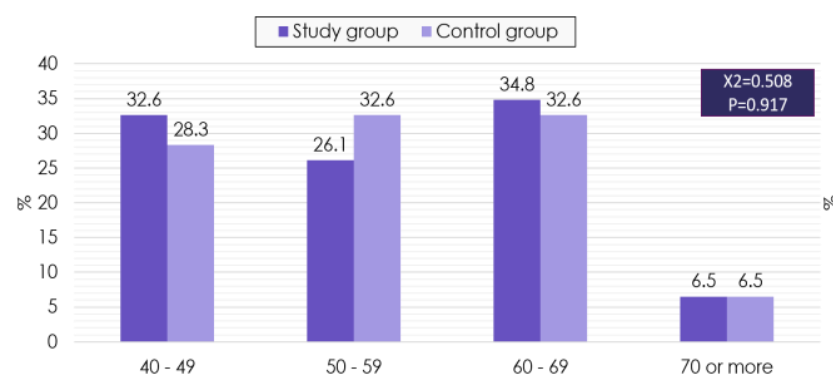

Figure 1. Age distribution within the two groups

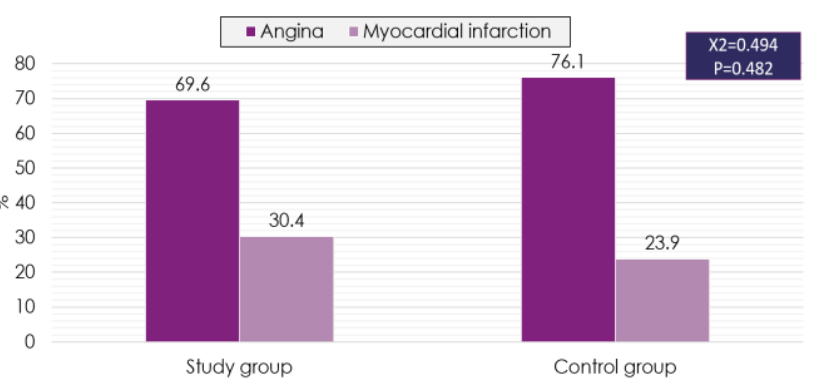

Figure 2. Distribution of patients 'diagnosis within the two groups

Table 2. Mean score of life-style practices among the studied patients $(n=92)$

\begin{tabular}{lllll}
\hline Life-style practices & $\begin{array}{l}\text { Study group }(\mathrm{n}=46) \\
\text { Mean } \pm \text { SD }\end{array}$ & $\begin{array}{l}\text { Control group }(\mathrm{n}=46) \\
\text { Mean } \pm \text { SD }\end{array}$ & $\begin{array}{l}\text { T test } \\
\mathrm{t}\end{array}$ & $\mathrm{p}$ \\
\hline Smoking & $9.1 \pm 3.7$ & $8.9 \pm 3.8$ & 0.306 & 0.761 \\
Eating unhealthy foods & $15.5 \pm 5.6$ & $14.4 \pm 6.0$ & 0.883 & 0.380 \\
Having unhealthy drinks & $9.0 \pm 3.5$ & $8.5 \pm 3.7$ & 0.633 & 0.529 \\
Practicing activities & $9.5 \pm 4.1$ & $8.5 \pm 3.4$ & 1.339 & 0.184 \\
Total score & $43.1 \pm 9.2$ & $40.3 \pm 8.2$ & 1.570 & 0.120 \\
\hline
\end{tabular}

*: Significant at $P \leq 0.05$ 
Table 3. Distribution of the two groups regarding physical function pre and post life style modification $(n=92)$

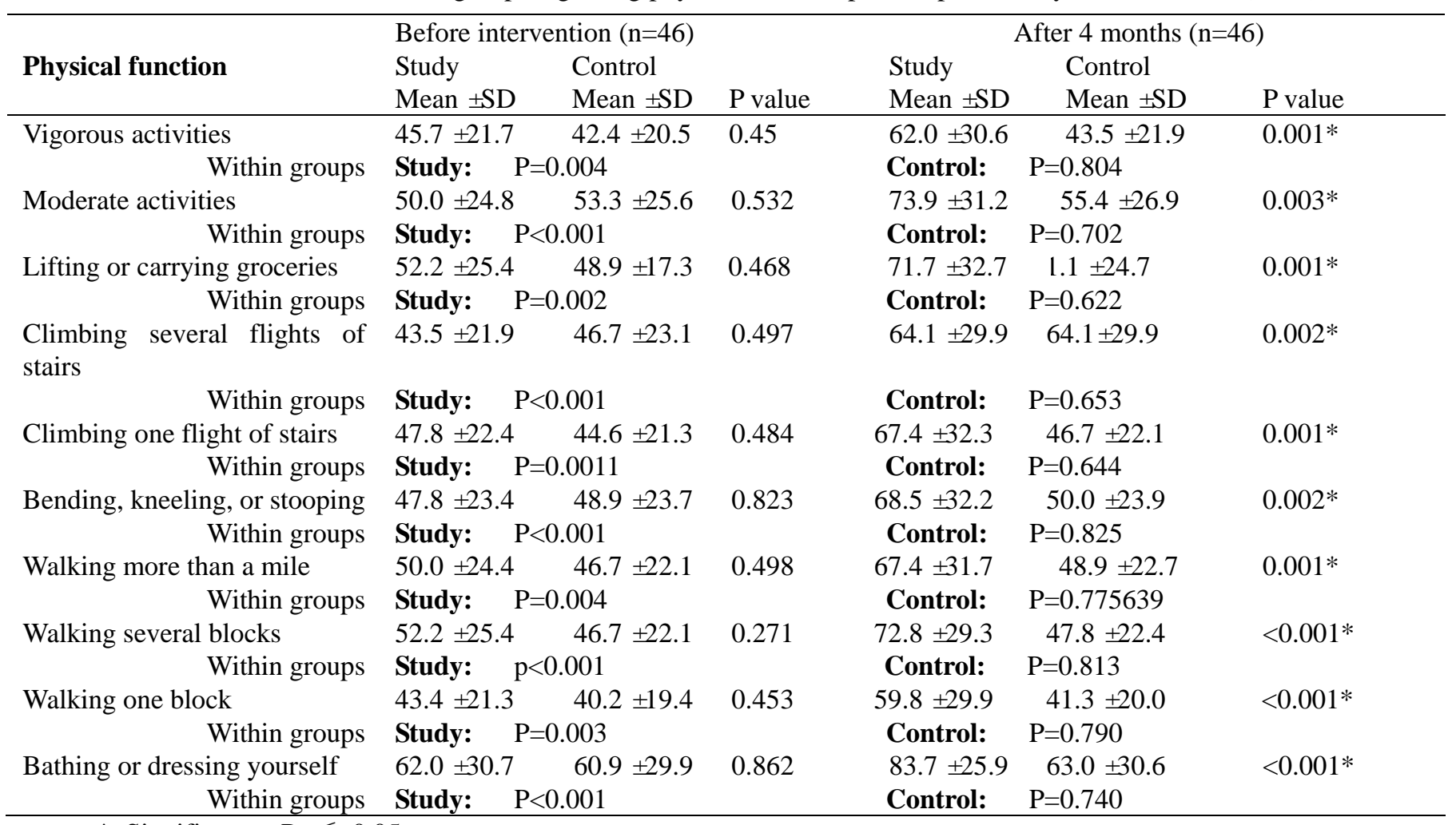

*: Significant at $\mathrm{P} \leqslant 0.05$

Table 4. Distribution of the two groups regarding role limitation due to physical problems, bodily pain and social function pre and post life style modification $(\mathrm{n}=92)$

\begin{tabular}{|c|c|c|c|c|c|c|}
\hline \multirow[b]{2}{*}{ Role Limitation due to } & \multicolumn{3}{|c|}{ Before intervention $(n=46)$} & \multirow[b]{2}{*}{$\begin{array}{l}\text { Study } \\
\text { Mean } \pm \text { SD }\end{array}$} & \multicolumn{2}{|c|}{ After 4 months $(\mathrm{n}=46)$} \\
\hline & $\begin{array}{l}\text { Study } \\
\text { Mean } \pm \text { SD }\end{array}$ & $\begin{array}{l}\text { Control } \\
\text { Mean } \pm \text { SD }\end{array}$ & $\mathrm{P}$ & & $\begin{array}{l}\text { Control } \\
\text { Mean } \pm \text { SD }\end{array}$ & $P$ value \\
\hline physical problems & & & & & & \\
\hline $\begin{array}{l}\text { Reduce time you spent on work or activities } \\
\text { Within groups }\end{array}$ & $\begin{array}{l}37.0 \pm 17.8 \\
\text { Study: }\end{array}$ & $\begin{array}{l}41.3 \pm 20.8 \\
\mathrm{P}<0.001\end{array}$ & 0.290 & $\begin{array}{l}65.2 \pm 31.2 \\
\text { Control: }\end{array}$ & $\begin{aligned} & 43.5 \pm 21.1 \\
\mathrm{P}= & 0.616\end{aligned}$ & $<0.001 *$ \\
\hline $\begin{array}{l}\text { Accomplished less than you would like } \\
\text { Within groups }\end{array}$ & $\begin{array}{l}39.1 \pm 19.3 \\
\text { Study: }\end{array}$ & $\begin{array}{l}37.0 \pm 17.8 \\
\mathrm{P}<0.001\end{array}$ & 0.589 & $\begin{array}{l}63.0 \pm 30.8 \\
\text { Control: }\end{array}$ & $\begin{aligned} & 39.1 \pm 19.3 \\
\mathrm{P} & =0.589\end{aligned}$ & $<0.001 *$ \\
\hline $\begin{array}{l}\text { Were limited in the kind of work or other } \\
\text { activities }\end{array}$ & $39.1 \pm 19.3$ & $41.3 \pm 20.8$ & 0.600 & $65.2 \pm 32.2$ & $43.5 \pm 21.1$ & $<0.001 *$ \\
\hline $\begin{array}{l}\text { Within groups } \\
\text { Had difficulty performing the work or other } \\
\text { activities }\end{array}$ & $\begin{array}{l}\text { Study: } \\
34.8 \pm 16.2\end{array}$ & $\begin{array}{l}\mathrm{P}<0.001 \\
32.6 \pm 15.4\end{array}$ & 0.506 & $\begin{array}{l}\text { Control: } \\
56.5 \pm 27.1\end{array}$ & $\begin{aligned} \mathrm{P}= & 0.616 \\
& 34.8 \pm 17.2\end{aligned}$ & $<0.001 *$ \\
\hline $\begin{array}{ll}\text { Bodily Pain } & \text { Within groups }\end{array}$ & Study: & $\mathrm{P}<0.001$ & & Control: & $\mathrm{P}=0.520$ & \\
\hline $\begin{array}{l}\text { How much bodily pain have you had? } \\
\text { Within groups }\end{array}$ & $\begin{array}{l}56.5 \pm 27.1 \\
\text { Study: }\end{array}$ & $\begin{array}{l}47.0 \pm 23.0 \\
\mathrm{P}=0.003\end{array}$ & 0.073 & $\begin{array}{l}71.7 \pm 20.9 \\
\text { Control: }\end{array}$ & $\begin{aligned} & 48.7 \pm 23.3 \\
\mathrm{P} & =0.726\end{aligned}$ & $<0.001 *$ \\
\hline $\begin{array}{l}\text { How much did pain interfere with your normal } \\
\text { work? }\end{array}$ & $54.3 \pm 25.4$ & $49.5 \pm 20.1$ & 0.309 & $66.8 \pm 19.8$ & $50.5 \pm 18.6$ & $<0.001 *$ \\
\hline 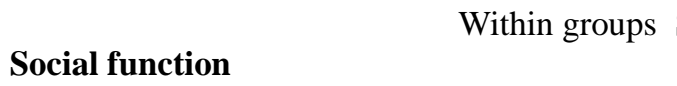 & Study: & $\mathrm{P}=0.01$ & & Control: & $\mathrm{P}=0.788$ & \\
\hline $\begin{array}{r}\text { Had difficulty participating in social activities } \\
\text { Within groups }\end{array}$ & $\begin{array}{l}55.4 \pm 26.3 \\
\text { Study: }\end{array}$ & $\begin{array}{l}48.4 \pm 23.4 \\
\mathrm{P}=0.005\end{array}$ & 0.181 & $\begin{array}{l}70.1 \pm 22.1 \\
\text { Control: }\end{array}$ & $\begin{array}{l}48.4 \pm 23.4 \\
\mathrm{P}=1.000\end{array}$ & $<0.001 *$ \\
\hline $\begin{array}{l}\text { How much physical health affected on social } \\
\text { activitie }\end{array}$ & $31.0 \pm 13.1$ & $30.7 \pm 14.7$ & 0.918 & $38.0 \pm 18.1$ & $30.7 \pm 14.7$ & $0.03 *$ \\
\hline Within groups & Study: & $\mathrm{P}=0.03$ & & Control: & $\mathrm{P}=1.000$ & \\
\hline
\end{tabular}

*: Significant at $P \leq 0.05$ 
Table 5. Distribution of the two groups regarding mental health and role limitations caused by emotional problems pre and post life style modification $(\mathrm{n}=92)$

\begin{tabular}{|c|c|c|c|c|c|c|}
\hline & \multicolumn{2}{|c|}{ Before intervention $(n=46)$} & \multicolumn{3}{|c|}{ After 4 months $(\mathrm{n}=46)$} & \multirow[b]{3}{*}{$\mathrm{P}$} \\
\hline & Study & Control & & Study & Control & \\
\hline & Mean \pm SD & Mean \pm SD & $\mathrm{P}$ & Mean \pm SD & Mean \pm SD & \\
\hline \multicolumn{7}{|l|}{ Mental Health } \\
\hline \multirow{2}{*}{$\begin{array}{l}\text { Have you been a very nervous person? } \\
\text { Within groups }\end{array}$} & $47.0 \pm 23.4$ & $55.2 \pm 20.7$ & 0.078 & $64.8 \pm 24.7$ & $54.8 \pm 20.8$ & $0.03 *$ \\
\hline & Study: & $\mathrm{P}<0.001$ & & Control: & $\mathrm{P}=0.920$ & \\
\hline \multirow{2}{*}{$\begin{array}{l}\text { Have you felt that nothing could cheer you up? } \\
\text { Within groups }\end{array}$} & $53.0 \pm 25.4$ & $55.7 \pm 22.3$ & 0.602 & $65.7 \pm 22.6$ & $54.8 \pm 22.9$ & $0.02 *$ \\
\hline & Study: & $\mathrm{P}=0.01$ & & Control: & $\mathrm{P}=0.854$ & \\
\hline \multirow{2}{*}{$\begin{array}{l}\text { You felt calm and peaceful? } \\
\text { Within groups }\end{array}$} & $47.8 \pm 23.5$ & $42.6 \pm 21.1$ & 0.267 & $59.6 \pm 28.7$ & $43.0 \pm 21.2$ & $0.002 *$ \\
\hline & Study: & $\mathrm{P}=0.03$ & & Control: & $\mathrm{P}=0.928$ & \\
\hline \multirow{2}{*}{$\begin{array}{l}\text { Have you felt downhearted and blue? } \\
\text { Within groups }\end{array}$} & $50.0 \pm 24.9$ & $56.5 \pm 22.0$ & 0.188 & $66.5 \pm 21.9$ & $56.1 \pm 22.2$ & $0.02 *$ \\
\hline & Study: & $\mathrm{P}=0.001$ & & Control: & $\mathrm{P}=0.925$ & \\
\hline \multirow{2}{*}{$\begin{array}{l}\text { Have you been a happy person? } \\
\text { Within groups }\end{array}$} & $49.6 \pm 24.2$ & $42.6 \pm 20.6$ & 0.156 & $61.3 \pm 25.4$ & $43.0 \pm 20.7$ & $<0.001 *$ \\
\hline & Study: & $\mathrm{P}=0.02$ & & Control: & $\mathrm{P}=0.926$ & \\
\hline \multicolumn{7}{|c|}{ Role limitations caused by emotional problems } \\
\hline \multirow{2}{*}{$\begin{array}{l}\text { Reduce time you spent on work or activities } \\
\text { Within groups }\end{array}$} & $41.3 \pm 20.5$ & $39.1 \pm 19.3$ & 0.598 & $63.0 \pm 31.8$ & $39.1 \pm 19.3$ & $<0.001 *$ \\
\hline & Study: & $\mathrm{P}<0.001$ & & Control: & $\mathrm{P}=1.000$ & \\
\hline \multirow{2}{*}{$\begin{array}{l}\text { Accomplished less than you would like } \\
\text { Within groups }\end{array}$} & $41.3 \pm 19.8$ & $39.1 \pm 19.3$ & 0.591 & $63.0 \pm 28.8$ & $39.1 \pm 19.3$ & $<0.001 *$ \\
\hline & Study: & $\mathrm{P}<0.001$ & & Control: & $\mathrm{P}=1.000$ & \\
\hline \multirow{2}{*}{$\begin{array}{c}\text { Didn't do work or other activities as carefully as } \\
\text { usual }\end{array}$} & $32.6 \pm 15.4$ & $34.8 \pm 16.2$ & 0.506 & $56.5 \pm 27.1$ & $34.8 \pm 17.2$ & $<0.001 *$ \\
\hline & Study: & $\mathrm{P}<0.001$ & & Control: & $\mathrm{P}=1.000$ & \\
\hline
\end{tabular}

*: Significant at $P \leq 0.05$

Table 6. Distribution of the two groups regarding energy/fatigue and general health pre and post life style modification $(\mathrm{n}=92)$

\begin{tabular}{|c|c|c|c|c|c|c|}
\hline & \multicolumn{2}{|c|}{ Before intervention $(n=46)$} & \multicolumn{4}{|c|}{ After 4 months $(n=46)$} \\
\hline & Study & Control & & Study & Control & \\
\hline & Mean \pm SD & Mean \pm SD & $\mathrm{P}$ & Mean \pm SD & Mean \pm SD & P value \\
\hline \multicolumn{7}{|l|}{ Energy/Fatigue } \\
\hline Did you feel full of pep? & $50.0 \pm 24.2$ & $43.5 \pm 21.3$ & 0.175 & $61.3 \pm 19.0$ & $43.48 \pm 21.3$ & $<0.001 *$ \\
\hline Within groups & Study: & $\mathrm{P}=0.01$ & & Control: & $\mathrm{P}=1.000$ & \\
\hline \multirow{2}{*}{$\begin{array}{l}\text { Did you have a lot of energy? } \\
\text { Within groups }\end{array}$} & $50.4 \pm 25.1$ & $43.0 \pm 20.5$ & 0.125 & $61.3 \pm 20.0$ & $43.9 \pm 20.1$ & $<0.001^{*}$ \\
\hline & Study: & $\mathrm{P}=0.024$ & & Control: & $\mathrm{P}=0.832$ & \\
\hline Did you feel worn out? & $50.4 \pm 24.6$ & $5 \quad 58.7 \pm 23.6$ & 0.102 & $67.8 \pm 22.9$ & $57.8 \pm 24.3$ & $0.04 *$ \\
\hline Within groups & Study: & $\mathrm{P}<0.001$ & & Control: & $\mathrm{P}=0.862$ & \\
\hline \multirow[t]{2}{*}{ Did you feel tired? } & $48.3 \pm 23.5$ & $47.4 \pm 22.9$ & 0.853 & $59.1 \pm 22.3$ & $48.3 \pm 23.4$ & $0.02 *$ \\
\hline & Study: & $\mathrm{P}=0.02$ & & Control: & $\mathrm{P}=0.853$ & \\
\hline \multicolumn{7}{|l|}{ General health } \\
\hline $\begin{array}{l}\text { I seem to get sick a little lesser than } \\
\text { other people }\end{array}$ & $31.5 \pm 13.8$ & $36.4 \pm 17.5$ & 0.139 & $48.4 \pm 22.6$ & $37.0 \pm 17.5$ & $0.008^{*}$ \\
\hline \multirow{2}{*}{$\begin{array}{l}\text { Within groups } \\
\text { I am as healthy as anybody I know }\end{array}$} & Study: & $\mathrm{P}<0.001$ & & Control: & $\mathrm{P}=0.870$ & \\
\hline & $60.9 \pm 27.2$ & $62.0 \pm 19.5$ & 0.826 & $71.7 \pm 23.9$ & $61.4 \pm 19.5$ & $0.02 *$ \\
\hline Within groups & Study: & $\mathrm{P}=0.04$ & & Control: & $\mathrm{P}=0.894$ & \\
\hline \multirow{2}{*}{$\begin{array}{l}\text { I expect my health to get better } \\
\text { Within groups }\end{array}$} & $34.2 \pm 15.2$ & $35.3 \pm 16.3$ & 0.739 & $45.7 \pm 22.3$ & $35.9 \pm 16.4$ & $0.01 *$ \\
\hline & Study: & $\mathrm{P}=0.005$ & & Control: & $\mathrm{P}=0.874$ & \\
\hline \multirow[t]{2}{*}{ My health is excellent } & $62.0 \pm 27.3$ & $60.9 \pm 22.1$ & 0.834 & $72.3 \pm 19.2$ & $60.3 \pm 22.1$ & $0.007 *$ \\
\hline & Study: & $\mathrm{P}=0.03$ & & Control: & $\mathrm{P}=0.906$ & \\
\hline
\end{tabular}

*: Significant at $P \leq 0.05$ 
Table 7. Distribution of the two groups regarding total health status score pre and post life style modification $(\mathrm{n}=92)$

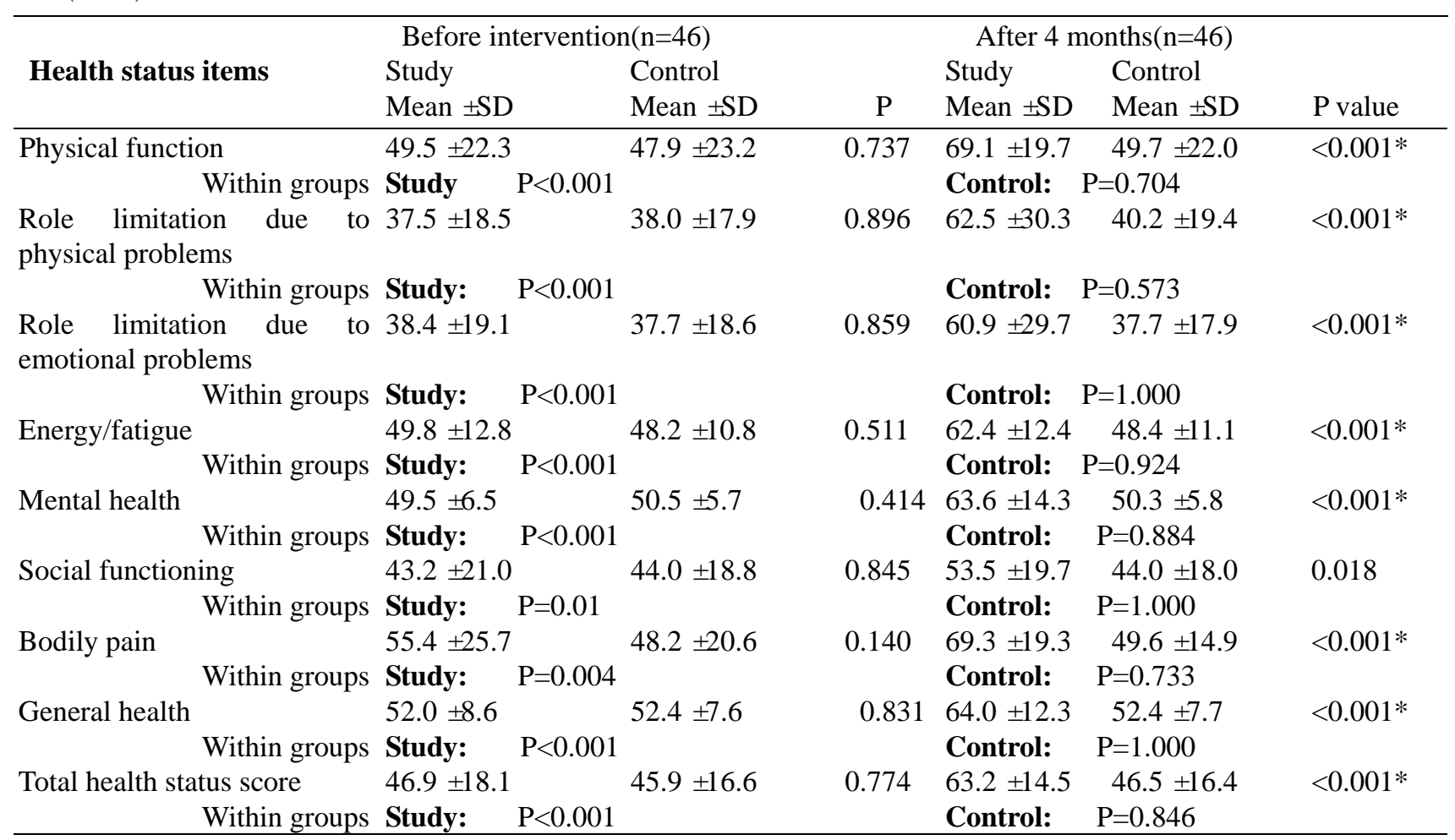

*: Significant at $P \leq 0.05$

\section{Discussion}

\subsection{Coronary Heart Disease Is the Primary Cause of Mortality Worldwide}

Cardiovascular diseases is accountable for about $10 \%$ of the total load of a disease every year in low- and middle-income countries and 18\% in high income countries (World Health Organization, 2013). In the Middle East, there is a fairly elevated mortality from CVD. The age-standardized cardiovascular death rates in countries as Iraq, Yemen, Egypt, Lebanon, and Jordan, are two times higher than the proportional figures for the United States. About 25-40\% of deaths in several Arab countries are due to cardiovascular diseases. Which, these risk factors contribute to the growing burden of strokes, heart failure, MI and peripheral vascular disease (Shantouf, 2012).

5.2 Reviewing socio-demographic characteristics of the study sample of the present study revealed that, the studied patients from the study and control group were homogenous as regard to their socio-demographic characteristics where they had no significant statistical differences. These results are consistent with Salameh, et al. (2012) who reported similarity in the intervention and control groups regarding their socio-demographic data.

5.3 Smoking cessation is the most valuable and the most gainful factor of all the lifestyle modification intervention that suggested to prevent CAD, the current study showed serious findings, as the studied patients from both groups had low scores regarding smoking practices. This finding was in the same line with a study done by Aslanabadi, et al. (2008) showed that prior applying a lifestyle modification intervention, 30\% of the patients in the study group and in the usual care group were smokers. Also, Dehghani, et al., (2015) noted that more than half of the study participants were smokers at baseline assessment.

5.4Extreme caffeine intake is a risk factor for CAD, however it still practiced by CAD patients. This fact was reported in the present study, as the study participants from both groups had low scores regarding having unhealthy drinks, as tea, coffee and fatty drinks this may be referred to the egyptian culture where they consider tea and coffee the popular drinks in Egypt. This finding was in the same line with (Abd-Elmawlaa, 2010), who found that majority of the sample (less than two-third) were drinking tea and coffee and didn't want to stop them. Regarding salt and fatty food intake, the current study demonstrated that the study participants from both groups had low scores regarding eating unhealthy food as salty and fatty foods, this may be attributable to eating habits of egyptian 
people that depending mainly in preparing meals containing frying foods, which is congruent with Khalife-Zadeh, Dorri, \& Shafiee (2015) who noted that their study participants had hyperlipidemia. In addition Safabakhsh, et al.(2016), conducted a study to determine the impact of a health promotion program on behavior in terms of the dimensions of the health promoting lifestyle profile in patients after coronary artery bypass graft, they discovered that the majority of the study participants were eating fatty foods. According to the present study the study participants from both groups had low scores regarding practicing activities like walking, physical exercise, and recreational activity this may be due to the presence of technology in our life that permits subjects to do most of their tasks without movement. These results are confirmed by the findings of the previous studies (Young, 2005; Cox, 2007 \& Maddison et al, 2014) who noted reduction among main part of participants concerning practice of exercises and recreational activities. Furthermore, Safabakhsh, et al. (2016), concluded that, the majority of participants in the experimental and control groups had a sedentary lifestyle and didn't perform exercises.

5.5 There was statistically significant difference after four months of intervention between the study and control groups regarding physical function, and role limitation due to physical problems, this may be related to the positive effect of activity on improvement of the circulation and strength of the cardiac muscle. In this regard, it has been shown that unsuccessful behavior change related to physical restrictions had a harmful effect on the progress of ischemic heart disease patients (Peterson et al., 2010). While, exercise have to be sustained for long-term to achieve the enhancement (Herdy, Marcchi \& Vila, 2008). Moreover, patients' hard work tolerance advanced extensively with exercise performance (Giallauria et al, 2008). That is consistent with, Salameh, et al. (2012), found that, the initial assessment of physical activity established a similarity between the two groups and improvement in the intervention group after education. Moreover Dehghani, et al., (2015) showed a considerable raise in physical activity in the study group as compared to the control group after six months to baseline data. These findings are in contrast to Eshah, (2011) who reported that patients have little scores in the physical function result from the shortage in informational programs and rehabilitation services.

5.6 There is a significant enhancement in the pain sensation among the study group post four months of life-style modification intervention as reported by the results of the current study. This finding is congruent with Saeidi, et al.,(2013), who discovered a significantly progress in bodily pain scores among the study participants compared to baseline scores. While this result is inconsistent with Yu et., al. (2004) who demonstrated that the intervention group had better scores in all domains of life style modifications, but differences between groups were no longer significant. There were no longer any statistically significant differences between groups in the percentage of participants complaining chest pain, and deterioration of chest pain.

5.7 The social position of the patients is a very important parameter that must be taken in mind when organizing a multidisciplinary intervention, in this regard, this study showed that, there was statistically significant difference after four months of intervention between the study and control groups. This is in accordance with Tofighi, et al., (2012) who showed that it was a statistically significant upgrading in the study group for social functioning post intervention. In the same line, Khalife-Zadeh, Dorri and Shafiee (2015), showed that an insignificant difference was found between the study and control groups in all aspects except for social function favoring of the study group. On the other hand Mohammadi et al., (2006) \& Antonakoudis, et al. (2006) who studied impact of rehabilitation on quality of life among patients with myocardial infarction, they reported that cardiac rehabilitation did not modify social domain of quality of life.

5.8 This study showed that, there was statistically significant difference after four months of intervention between the study and control groups regarding their mental health. This finding may be attributable to that improvement of physical health can decrease stressors and elevate patients' morale which in turn enhance their mental health. This finding is consistent with Sandvik, Seim, \& Vanvik, (2000) who showed that cardiac rehabilitation resulted in development in all dimensions of life style modifications of the study group. This goes in the same line with an investigation by Grace, et al., (2008) showed that mental health and anxiety were enhanced post cardiac rehabilitation. Another systematic review indicated that cardiac rehabilitation can diminish psychological stress of coronary disorders Taylor, et al., (2010). In addition, Samartzis, et al., (2013) in their meta-analysis study, revealed that cardiac rehabilitation improved participants' mental and psychological health.

5.9 Concerning role limitations caused by emotional problems, this study indicated that, there was statistically significant difference after four months of intervention between the study and control groups. This can be interpreted as role limitations caused by CAD, which may influence patients' ability to work is frankly influence patients' capability to carry out tasks allied to daily life activities. This is in accordance with Rumsfeld, Magid, \& Plomondon. (2003), who recommended that all post AMI patients have to participate in CR programs including group or team intervention. This approach increases the patient's confidence and well-being feeling. Chodosh, et al.( 2005), added that coronary syndrome may also result in emotional impaired functioning due to feelings 
associated with fears from death and losing families (i.e. inability to maintain family commitments and relationships) As well as Zwisler, et al (2008) revealed that health status was better post cardiac rehabilitation further more communication and work / leisure activities were also absolutely progressed. This progress is valuable to declare as it is a vital element of health status.

5.10 The finding of the current study reported a significant improvement in energy/fatigue and general health of the study group compared to the control group after four months from life-style modification intervention These results are compatible with the findings of Salameh, et al. (2012) who found an important advance in energy/fatigue and general health of coronary artery disease patients post cardiac rehabilitation program. Also Saeidi, et al. (2013), noted the same results. This is contradicted Yu et al. (2004) who reported that evaluation of general health dimension in two groups indicated no significant difference. Furthermore, Khalife-Zadeh, Dorri, and Shafiee (2015), noted that an important discrepancy was established between the study and control groups in all aspects of health status except for general health and social function in favor of the study group.

5.11 The total health status of coronary artery disease patients is significantly improved after four months from lifestyle modification intervention as evidenced by the results of the present study. An important possible explanation for the improvement of lifestyle modification intervention on health status of coronary artery disease patients in the present study is the significant improvement in the level of knowledge regarding CAD patients after the application of the educational intervention. This is congruent with the findings of prior studies (Meischke et al., 2000\& Lunelli et al., 2009) that found a positive association between knowledge and compliance to healthy lifestyle. In the same line, Attarbashi-Moghadam et al., (2014) confirmed that a progress was recognized in total health status. According to three studies measured health status, three studies finding indicated verification for enhancement Devi, Powell, \& Singh, (2014) \& Maddison et al, (2014) \& Varnfield et al, (2014).

\section{Conclusion}

The results of the current study concluded that life style modification intervention for coronary artery disease patients is an effective method that can improve their health status in the aspects of physical function, bodily pain, social function, role limitation due to physical or emotional problems and mental health.

\section{Recommendations}

Based upon results of the current study, the following recommendations were suggested:

- Further research studies are necessary to verify the effect of intensified lifestyle modification intervention on cardiovascular risk factors, principally atherosclerosis and blood pressure.

- Patients` education should be held in continuous manner, because it is a fundamental part of their treatment.

- $\quad$ Level of patients`adherence to healthy lifestyle should be assessed over time.

- Cardiac rehabilitation centers should be established and encouraged.

- $\quad$ Additional educational programs for patients and health care giver are required about risk factors and preventive methods of coronary artery diseases.

\section{References}

Abd-Elmawlaa, R.A. (2010). Assess compliance to theraputic regimen for patients with angina pectoris. Master Thesis, Medical Surgical Nursing, Faculty of Nursing, Mansoura University.

Ahmad, A., \& Tawalbeh, L. (2015). Patients with coronary artery disease in the north of Jordan: toward healthy lifestyle intervention. International Journal of Public Health Research, 3(5), 279-287.

Antonakoudis, H., Kifnidis, K., Andreadis, A., Fluda, E., Konti, Z., Papagianis, N., ... Poulimenos, L. (2006). Cardiac rehabilitation effects on quality of life in patientsafter acute myocardial infarction. Hippokratia, 10(4), 176-181.

Aslanabadi, N., Shahamfar, J., Kumar, G.V., Hadi, H.S., Saeed, D., Mohamadreza, S., \& Hossein, M.A. (2008). Cardiac risk factor changes through an intensive multifactorial life style modification program in CHD patients: results from a two year follow up. $J$ Biol Sci, 8(2), 248-257. https://doi.org/10.3923/jbs.2008.248.257

Attarbashi-Moghadam, B., Hadian, M., Baqeri, H., Tavakol, K., Salarifar, M., \& Jalaie, S. (2014). The effects of Phase II cardiac rehabilitation on quality of life scales in post coronary artery bypass grafts patients. Modern Rehabil, 12, 12-18. 
Brinks, J., Fowler, A., Franklin, B.A., \& Dulai, J. (2017). Lifestyle Modification in Secondary Prevention: Beyond Pharmacotherapy. American Journal of Life Style Medicine, 11(2). https://doi.org/10.1177/1559827616651402

Chodosh, J., Morton, S.C., Mojica, W., Maglione, M., Suttorp, M.J., Hilton, L., ... Shekelle, P. (2005, September). Meta-analysis: Chronic disease self-management programs for older adults. Ann Intern Med., 143(6), 427-438 https://doi.org/10.7326/0003-4819-143-6-200509200-00007

Coster, S., \& Norman, I. (2009). Cochrane reviews of educational and self-management interventions to guide nursing practice: a review. International Journal of Nursing Studies, 46, 508-528. https://doi.org/10.1016/j.ijnurstu.2008.09.009

Cox, R.H. (2007). Sport psychology: Concepts and applications (6th ed.). New York, NY: McGraw Hill.

Dehghani, A., Bhasin, S.K., Dwivedi, S., \& Malhotra, R.K. (2015). Influence of Comprehensive Life Style Intervention in Patients of CHD. Global Journal of Health Science, 7(7). Canadian Center of Science and Education.

Devi, R., Powell, J., \& Singh, S. (2014). A web-based program improves physical activity outcomes in a primary care angina population: randomized controlled trial. Journal of Medical Internet Research, 16(9), e186.

Eshah, N., Bond, E., \& Froelicher, E. (2010). The effects of cardiovascular disease prevention program on knowledge and adoption of a heart healthy lifestyle in Jordanian working adults. European Journal of Cardiovascular Nursing, 9, 244-253.

Eshah, N.F. (2011). Jordanian acute coronary syndrome patients' learning needs: Implications for cardiac rehabilitation and secondary prevention programs. Nursing \& Health Sciences, 13(3), 238-245.

Framingham Heart Study. (2016). A project of the National Lung and Heart and Blood Institute and Boston University. History of the Framingham Heart Study. Retrieved from http://www.framinghamheartstudy.org/about-fhs/history.php

Fredericks, S., Beanlands, H., Spalding, K., \& Da Silva, M. (2010). Effects of the characteristics of teaching on the outcomes of heart failure patient education interventions: A systematic review. European Journal of Cardiovascular Nursing, 9(1), 30-37. https://doi.org/10.1016/j.ejcnurse.2009.08.002

Giallauria, F., Cirillo, P., Lucci, R., Pacileo, M., De Lorenzo, A., \& D’Agostino, M. (2008). Left ventricular remodelling in patients with moderate systolic dysfunction after myocardial infarction: favourable effects of exercise training and predictive role of N-terminal pro-brain natriuretic peptide. European Journal of Cardiovascular Prevention and Rehabilitation, 15(1), 113-118. https://doi.org/10.1097/HJR.0b013e3282f00990

Go, A.S., Mozaffarian, D., Roger, V.L., Benjamin, E.J., Berry, J.D., Blaha, M.J., \& Turner, M.B. (2014). Heart disease and stroke statistics: A report from the American Heart association. Circulation, 129, e28-e292. https://doi.org/10.1161/01.cir.0000441139.02102.80

Grace, S.L., Grewal, K., Arthur, H.M., Abramson, B.L., \& Stewart, D.E. (2008). A prospective, controlled multisite study of psychosocial and behavioral change following women's cardiac rehabilitation participation. J Womens Health (Larchmt), 17(2), 241-248. https://doi.org/10.1089/jwh.2007.0519

Herdy, A.H., Marcchi, P.L., \& Vila, A. (2008). Pre- and postoperative cardiopulmonary rehabilitation in hospitalized patients undergoing coronary artery bypass surgery: a randomized controlled trial. Am J Phys Med Rehabi, 87(9), 714-719. https://doi.org/10.1097/PHM.0b013e3181839152

Khalife-Zadeh, A., Dorri, S., \& Shafiee, S. (2015, Sep-Oct.). The effect of cardiac rehabilitation on quality of life in patients with acute coronary syndrome. Iran J Nurs Midwifery Res., 20(5), 588-593.

Libby, R., Bonow, H., Zipes, E., \& Mann, N. (2008). Braunwald's heart disease (8th ed.). A textbook of cardiovascular. Medicine. Philadelphia: W. B. Saunders, 1353-1358.

Lourenço LB de, A., Rodrigues, R.C.M., São-João, T.M., Gallani, M.C., \& Cornélio, M.E. (2015). Quality of life of coronary artery disease patients after the implementation of planning strategies for medication adherence. Revista Latino-Americana de Enfermagem, 23(1), 11-19. https://doi.org/10.1590/0104-1169.0144.2519

Lunelli, R.P., Portal, V.L., Esmero, F.G., Moraes, M.A., \& de Souza, E.N. (2009). Patients with coronary arterial diseases adherence to pharmacological and nonpharmacological therapy. Acta Paul Enferm, 22, 367-373. https://doi.org/10.1590/S0103-21002009000400003 
Maddison, R., Pfaeffli, L., Whittaker, R., Stewart, R., \& Kerr AJiang, Y. (2014). A mobile phone intervention increases physical activity in people with cardiovascular disease: Results from the HEART randomized controlled trial. European Journal of Preventive Cardiology, 22(6), 701-709. https://doi.org/10.1177/2047487314535076

Meischke, H., Sellers, D.E., Robbins, M.L., Goff, D.C., Daya, M.R., Meshack, A., \& Hand, M.M (2000). Factors that influence personal perceptions of the risk of an acute myocardial infarction. Behavioral Medicine, 26(1), 4-13. https://doi.org/10.1080/08964280009595748

Mohammadi, M., Taherian, A., Hoseini, M., \& Rahgozar, M. (2006). Effects of home-based cardiac rehabilitation on quality of life in patients with myocardial infarction. Rehabilitation, 7(3),11-19.

Peterson, J.C., Allegrante, J.P., Pirraglia, P.A., Robbins, L., Lane, K.P., Boschert, K.A., \& Charlson, M.E. (2010). Living with heart disease after angioplasty: A qualitative study of patients who have been successful or unsuccessful in multiple behavior change. Heart \& Lung: The Journal of Acute and Critical Care, 39(2), 105-115. https://doi.org/10.1016/j.hrtlng.2009.06.017

Roger, V.L., Go, A.S., Lloyd-Jones, D.M., Benjamin, E.J., \& Berry, J.D. (2012). Heart Disease and Stroke Statistics: A Report from the American Heart Association; Retrieved from http://circ.ahajournals.org/content/early/2011/12/15/CIR.0b013e31823ac046.citation.

Rumsfeld, J.S., Magid, D.J., \& Plomondon, M.E. (2003). History of depression, angina, and quality of life after acute coronary syndromes. Am Heart J., 145, 493-499. https://doi.org/10.1067/mhj.2003.177

Saeidi, M., Mostafavi, S., Heidari, H., \& Masoudi, S. (2013). Effects of a comprehensive cardiac rehabilitation program on quality of life in patients with coronary artery disease. ARYA Atheroscler, 9(3), 179-185. https://doi.org/10.1136/heartjnl-2013-304613.387

Safabakhsh, L., Arbabisarjou, A., Jahantigh, M., Nazemzadeh, M., Rigi, S.N., \& Nosratzehi, S. (2016). The Effect of Health Promoting Programs on Patient's Life Style After Coronary Artery Bypass Graft-Hospitalized in Shiraz hospitals. Global Journal of Health Science, 8(5), 154-159.

Salameh, B., Gomaa, N., El-Senousy, T., \& Salameh, O (2012). Effect Of An E-learning Program On The Quality Of life Of Patients With Coronary Heart Disease. Procedia-Social and Behavioral Sciences, 55, 284-293.

Samartzis, L., Dimopoulos, S., Tziongourou, M., \& Nanas, S. (2013). Effect of Psychosocial Interventions on Quality of Life in Patients With Chronic Heart Failure: A Meta-analysis of Randomized Controlled Trials. J. Card Fail, 19, 125-134.

Sandvik, H., Seim, A., \& Vanvik, A. (2000). A severity index for epidemiological surveys of female urinary incontinence: Comparison with 48-hour pad weighing tests. Neurourol. Urodyn, 19(2), 137-145.

Shantouf, R. (2012). Risk factors and the growing burden of cardiovascular disease in the Arab world, Cardiology. Clin Risk Manag, 4(8), 65-72.

Smith, S., Benjamin, E.J., Bonow, R.O., Braun, L.T., Creager, M.A., Franklin, B.A., \& Taubert, K.A. (2011). AHA/ACCF Secondary prevention and risk reduction therapy for patients with coronary and other atherosclerotic vascular disease. A Guideline from the American Heart Association and American College of Cardiology Foundation. Circulation, 124, 2458-2473.

Taylor, R.S., Dalal, H., Jolly, K., Moxham, T., \& Zawada, A. (2010). Home-based versus centre-based cardiac rehabilitation. Cochrane Database Syst Rev, (1), CD007130. https://doi.org/10.1002/14651858.CD007130.pub2

Timby, K.B., \& Smith, E.N. (2010). Introductory Medical Surgical Nursing, Caring for Clientts with Disorders of Coronary and Preipheral Blood Vessels (10th ed.). Philadelphia: Lippincott Williams \& Wilkins.

Tofighi, S., Kiadaliri, A.A., Sadeghifar, J. Raadabadi, M., \& Mamikhani, J. (2012). Health-Related Quality of Life among Patients with Coronary Artery Disease: A Post-Treatment Follow-Up Study in Iran. Cardiol Res Pract., 973-974. https://doi.org/10.1155/2012/973974

United States National Library of Medicine. (2016). Coronary heart disease. Retrieved from https://WWW.nlm.nih.gov/medlineplus/ency/article/007115.htm

Varnfield, M., Karunanithi, M., Lee, C.K., Honeyman, E., Arnold, D., \& Ding, H. (2014). Smartphone-based home care model improved use of cardiac rehabilitation in post myocardial infarction patients: results from a randomised controlled trial. Heart, 100(22), 1770-1779. https://doi.org/10.1136/heartjnl-2014-305783 
Wachira, J.K., \& Stys, T.P. (2013). Cardiovascular disease and bridging the diagnostic gap. South Dakota Medicine, 66(9), 366-369.

Ware, J.E., \& Sherbourne, C.D. (1992). The MOS 36-item short form health survey (SF-36). I. Conceptual framework and item selection. Medical Care, 30(6), 473-483. https://doi.org/10.1097/00005650-199206000-00002

Weberg, M., Hjermstad, M., Hilmarsen, C., \& Oldervoll, L. (2013). Inpatient cardiac rehabilitation and changes in self-reported health related quality of life-A pilot study. Annals of Physical and Rehabilitation Medicine. https://doi.org/10.1016/j.rehab

World Health Organization. (2014). World Bank, UNESCO, CIA and individual country databases for global health and causes of death. Retrieved from www.worldlifeexpectancy.com/egypt-coronary-heart-disease

World Health Organization. (2017). Cardiovascular diseases (CVDs) Fact sheet, Media Centre. Retrieved from http://www.who.int/mediacentre/factsheets/fs317/en

World Health Organization: Cardiovascular disease. (2013). Retrieved from http://www.who.int/cardiovasculardiseases/en/

Young, T.D. (2005, January/February). Factors determining exercise adherence. American Fitness. SPORT discus database.

Yu, C.M., Lau, C.P., Chau, J., McGhee, S., Kong, S.L., \& Cheung, B.M. (2004). A short course of cardiac rehabilitation program is highly cost effective in improving long-term quality of life in patients with recent myocardial infarction or percutaneous coronary intervention. Arch Phys Med Rehabil, 85, 1915-1922. https://doi.org/10.1016/j.apmr.2004.05.010

Zwisler, A.D., Soja, A.M., Rasmussen, S., Frederiksen, M., Abedini, S., \& Appel, J. (2008). Hospital-based comprehensive cardiac rehabilitation versus usual care among patients with congestive heart failure, ischemic heart disease, or high risk of ischemic heart disease: 12-month results of a randomized clinical trial. Am Heart J, 155(6), 1106-1113. https://doi.org/10.1016/j.ahj.2007.12.033

\section{Copyrights}

Copyright for this article is retained by the author(s), with first publication rights granted to the journal.

This is an open-access article distributed under the terms and conditions of the Creative Commons Attribution license (http://creativecommons.org/licenses/by/4.0/). 